\title{
Communication breakdown
}

DOI:

10.1038/nrmicro1786
Current treatments for bacterial infection depend on antibiotics that interfere with microbial growth and viability, resulting in the inevitable development of drug resistance and the demise of their effectiveness. One approach to overcome this problem is to develop anti-infective compounds that selectively disrupt pathways that mediate virulence without affecting microbial cell viability. New research published in PLoS Pathogens embraces this tactic by identifying a series of compounds that specifically target a quorum sensing (QS) system that is used by Pseudomonas aeruginosa to regulate the expression of its virulence determinants.

QS is a highly regulated communication system that is used by numerous pathogens to initiate and coordinate the expression of their virulence-gene repertoire, and the disruption of the system has no impact on the viability of the microorganism. In $P$. aeruginosa, one key QS system that

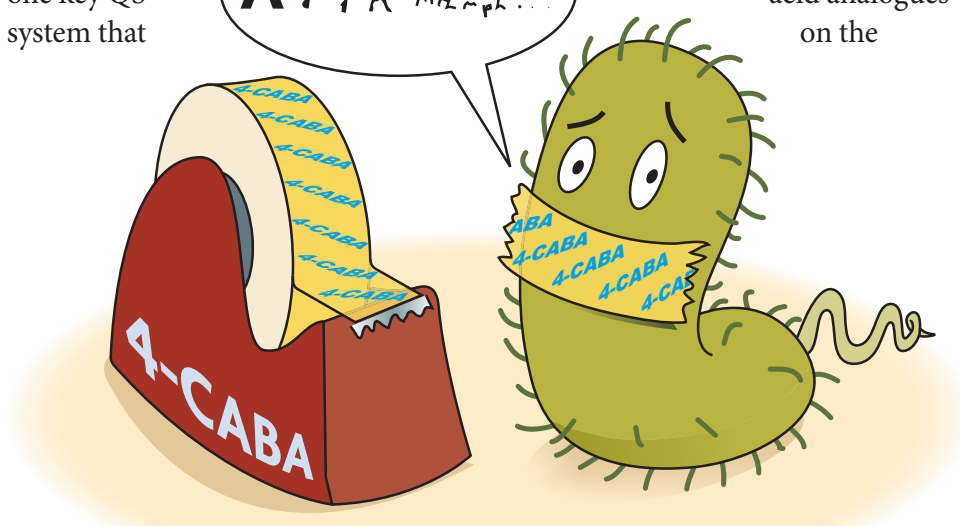

is crucial for virulence is the MvfRdependent QS regulatory pathway. After the transcriptional regulator MvfR is activated, it upregulates the expression of numerous virulence genes. The pathway is activated by the extracellular signals 4-hydroxy2-heptylquinoline (HHQ) and 3,4-dihydroxy-2-heptylquinoline (PQS), the syntheses of which ultimately depend on an anthranilic acid precursor. To inhibit this pathway, Laurence Rahme and colleagues focused on developing analogues of this precursor and identified three halogenated versions that specifically inhibited the biosynthesis of HHQ and PQS in both P. aeruginosa and Burkholderia thailandensis. By inhibiting the synthesis of these activating molecules, MvfR-dependent gene expression was disrupted, including factors such as hydrogen cyanide, chitinase, lectins and elastase. Lesic and colleagues also investigated the effect of these anthranilic acid analogues on the the production of key virulence virulence of $P$. aeruginosa in a mouse model of infection. Importantly, these compounds reduced both $P$. aeruginosa systemic dissemination and mortality in mice without impacting on bacterial viability at the site of infection. In addition, the authors observed that the ability of several bacterial pathogens, including $P$. aeruginosa, to tolerate salt was also compromised by the anthranilic acid analogues, which is an indication of the reduced ability of these microorganisms to withstand the hostile conditions of the host environment. These findings of increased osmosensitivity in several pathogens, together with the observation that the MvfR pathway is present in other microorganisms, suggest that these reagents have broad-spectrum activity against a range of clinically significant bacteria.

The increase in microorganisms that have developed resistance to currently available antimicrobial agents has become a major cause for concern worldwide. These findings provide a promising starting point in the journey to design and develop a new class of anti-infective agents that are effective against a wide range of human pathogens, and that have a reduced propensity to generate resistance.

David O'Connell

ORIGINAL RESEARCH PAPER Lesic, B. et al. Inhibitors of pathogen intercellular signals as selective anti-infective compounds. PLoS Pathog. 3, e126 (2007)

FURTHER READING Hentzer, M. \& Givskov M. Pharmacological inhibition of quorum sensing for the treatment of chronic bacterial infections. J. Clin. Invest. 112, 1300-1307 (2003) 